\title{
Growth modeling of human mandibles using non-Euclidean metrics
}

\author{
Klaus Baggesen Hilger*, Rasmus Larsen, Mark C. Wrobel \\ Informatics and Mathematical Modeling, Technical University of Denmark, Richard Petersens Plads, Building 321, DK-2800 Kgs. Lyngby, Denmark
}

\begin{abstract}
From a set of 31 three-dimensional computed tomography (CT) scans we model the temporal shape and size of the human mandible for analysis, simulation, and prediction purposes. Each anatomical structure is represented using 14851 semi-landmarks, and mapped into Procrustes tangent space. Exploratory subspace analyses are performed leading to linear models of mandible shape evolution in Procrustes space. The traditional variance analysis results in a one-dimensional growth model. However, working in a non-Euclidean metric results in a multimodal model with uncorrelated modes of biological variation related to independent component analysis. The applied non-Euclidean metric is governed by the correlation structure of the estimated noise in the data. The generative models are compared, and evaluated on the basis of a cross validation study. The new non-Euclidean analysis is completely data driven. It not only gives comparable results w.r.t. previous studies of the mean modeling error, but seems to better correlate to growth, and in addition provides the data analyst with alternative hypothesis of plausible shape evolution; hence aiding in the understanding of cranio-facial growth.
\end{abstract}

(c) 2003 Elsevier B.V. All rights reserved.

Keywords: Non-Euclidean metric; Maximum autocorrelation factors; Independent components; Minimum noise fractions; Active shape models

\section{Introduction}

Insight and understanding of facial growth is very important for pediatric cranio-facial surgeons. This paper is concerned with the mandible, the growth of which is particularly complex; due to the asynchronous teeth eruption and changes in the angular direction of the condylar process. The data are 31 mandibular surfaces acquired from computed tomography (CT) scans of a total of six subject with Apert syndrome. All scans were acquired for treatment and diagnostics purposes. In Apert syndrome, the mandible is not affected by the primary anomaly (Kreiborg et al., 1999), the data thus constitute a basis for modeling normal growth. Modeling of mandibular metamorphosis for analysis, simulation and prediction has implications for understanding the processes of bone growth and tooth eruptions. Processes that are not fully understood today.

\footnotetext{
*Corresponding author.

E-mail addresses: kbh@imm.dtu.dk (K.B. Hilger), http:// www.imm.dtu.dk/kbh (K.B. Hilger), rl@imm.dtu.dk http://
}

Furthermore, the models are directly applicable for craniofacial surgical planning in patients with severe congenital cranio-facial malformations.

The goal of this work is to model the temporal shape and size of the mandible. Subspace analyses are thus performed on the semi-landmark registered shapes projected into tangent space by a Procrustes analysis (Goodall, 1991; Dryden and Mardia, 1998). We extend the previous work by Andresen and Nielsen (2001), by analyzing the tangent space using a non-Euclidean metric. More specifically, we apply the maximum autocorrelation factors (MAF) analysis (Switzer and Green, 1984; Conradsen et al., 1985; Switzer and Ingebritsen, 1986; Hilger, 2001), instead of the principal components (PC) analysis (Hotelling, 1933). The applied MAF transform detects modes of variation with high degrees of landmark autocorrelation, whereas the PC analysis maximizes variance. The paradigm of MAF is thus that signal is not only characterized by high variance, but also by high correlation in the variation of neighboring homologous points. Related work using exploratory methods for decompositioning of biological shape data can be found in (Hilger et al., 2002; Larsen et al., 2002). 
The remaining paper consists of four sections. Section 2 introduces the data, the initial registration, establishment of correspondence, and alignment. Section 3 describes the applied statistical models, and summarize their results. Section 4 contains an evaluation of the applied methods in a cross-validation study predicting mandibular growth. In Section 5 we summarize and give some concluding remarks.

\section{Data material}

The data are CT scans of six subjects (four males and two females) with Apert syndrome. The subjects are scanned from three to seven times at ages between 1 month and 12 years, see Table 1 and Fig. 3(a) (subject versus age).

The mandibles are initially manually segmented from the CT scans of varying in-plane resolution down to 0.5 $\mathrm{mm}$. The surface extraction and registration is carried out using matching of the extremal mesh followed by a geometry-constrained diffusion (GCD) procedure described in (Andresen and Nielsen, 2001). Moreover, see (Paulsen and Hilger, 2003) on the relation between GCD and registration via Minimum Description Length analysis, (Davies et al., 2002), linked in a Markov random field framework. The resulting surfaces contains 14851 homologous points. The alignment of these semi-landmark registered shapes is obtained by applying a generalized (three-dimensional) Procrustes analysis (ten Berge, 1977). The shapes are thus aligned in a least-squares sense to a grand pole (i.e. the common mean mandible), maximizing the sum over all subject pair-wise correlations (Hilger, 2001).

Consider a set of $S$ shapes represented by $\left\{\boldsymbol{S}_{i}\right\}_{i=1}^{S}$, $S_{i} \in \mathbb{R}^{N \times k}$, where $N$ is the number of landmarks and $k$ the dimensionality of the shapes. Applying Euclidean similarity transformations $\left(T_{i}(\cdot), i=1, \ldots, S\right)$ consisting of scaling, rotation and translation, the Procrustes alignment maximizes the sum over all pair-wise correlations $R=$ $\sum_{i j} \operatorname{Corr}\left\{\operatorname{vec}\left(T_{i}\left(\boldsymbol{S}_{i}\right)\right), \operatorname{vec}\left(T_{j}\left(\boldsymbol{S}_{j}\right)\right)\right\}$. Let $\boldsymbol{S}_{i}^{P}=T_{i}\left(\boldsymbol{S}_{i}\right)$ represent the Procrustes aligned mandibles, then the average shape is given by $\overline{\boldsymbol{S}}=\Sigma_{i} \boldsymbol{S}_{i}^{P} / S$ shown in Fig. 1 .

Table 1

Age in month versus CT scan number for each patient

\begin{tabular}{lllllllll}
\hline & & \multicolumn{7}{c}{ Scan number } \\
\cline { 3 - 9 } Patient number & Sex & 1 & \multicolumn{1}{c}{2} & 3 & 4 & 5 & 6 & 7 \\
\hline 1 & M & 3 & 16 & 21 & 23 & 34 & & \\
2 & M & 1 & 7 & 23 & 54 & 56 & 60 & 72 \\
3 & M & 1 & 5 & 17 & 32 & 36 & & \\
4 & F & 3 & 27 & 46 & 62 & 131 & 132 & 144 \\
5 & M & 3 & 4 & 21 & 72 & & & \\
6 & F & 9 & 21 & 84 & & & & \\
\hline
\end{tabular}

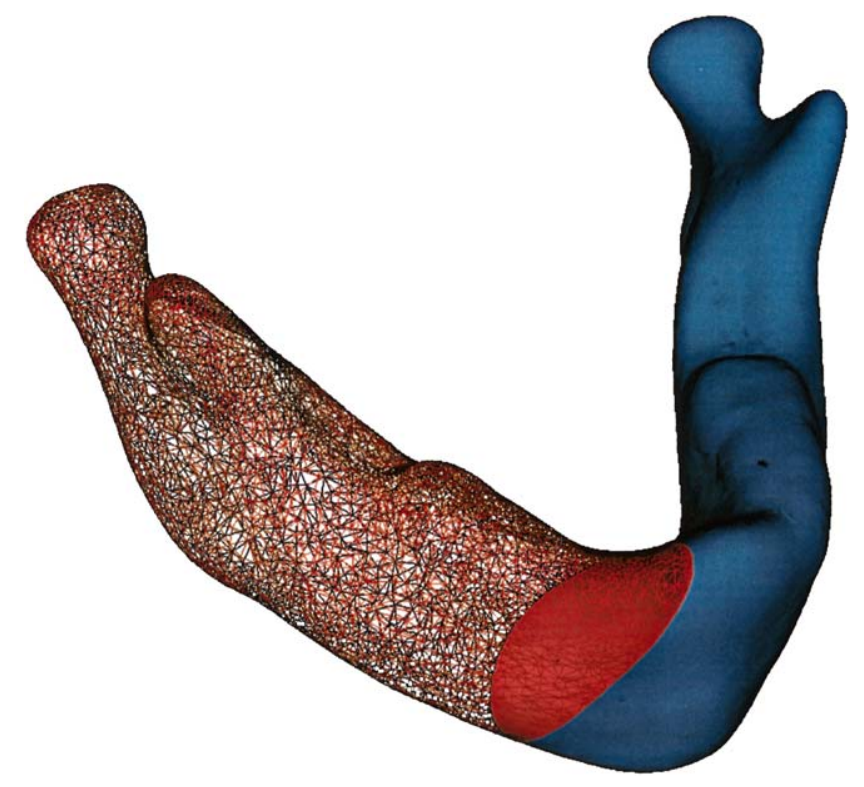

Fig. 1. The common mean mandible registered using 14851 vertices.

\section{Decomposition in Procrustes space}

\subsection{Principal components analysis}

We concatenate the set of $S$ shapes into a set of vectors $\left\{r_{i}=\operatorname{vec}\left(\boldsymbol{S}_{i}^{P}\right)\right\}_{i=1}^{S}$ with elements denoted by $r_{i}(\boldsymbol{x}), i=1$, $\ldots, S$, where $\boldsymbol{x}$ is a landmark index vector. Let $\boldsymbol{r}(\boldsymbol{x})=$ $\left[r_{1}(x) \ldots r_{S}(x)\right]^{\mathrm{T}}$ represent a random variable and assume first and second order stationarity, i.e. $E\{\boldsymbol{r}(\boldsymbol{x})\}=0$ and $D\{\boldsymbol{r}(\boldsymbol{x})\}=\boldsymbol{\Sigma}$. The PC transformation looks for Euclidean orthogonal modes of maximum variance. The basis for the PCs is identified as the conjugate eigenvectors of the dispersion matrix, $\boldsymbol{\Sigma}$. Let $\lambda_{1}=\operatorname{Var}\left\{\boldsymbol{a}_{1}^{\mathrm{T}} \boldsymbol{r}\right\} \geqslant \cdots \geqslant \lambda_{S} \geqslant 0$ be the eigenvalues with the corresponding conjugate eigenvectors $\boldsymbol{A}=\left[a_{1} \ldots a_{S}\right]$. Above, the variance decomposition is performed in Q-mode. Using the Eckart-Young's Theorem (Johnson, 1963), the eigenvectors for the R-mode solution becomes $\boldsymbol{B}=\left[\boldsymbol{b}_{1} \ldots \boldsymbol{b}_{S}\right]=\boldsymbol{R} \boldsymbol{\Lambda}^{-1 / 2} \boldsymbol{A}$, where $\boldsymbol{R}=\left[\boldsymbol{r}_{1} \ldots \boldsymbol{r}_{S}\right]$ and $\Lambda$ a diagonal matrix of the eigenvalues.

To first assess the inter-patient variability in the data the PC analysis is applied to the patient specific poles projected into tangent space using the grand mean pole, and results in a five dimensional subspace. The angular deviation of the patient specific poles from the grand pole are $6.3,6.1,6.9,6.9,6.6$ and $6.3^{\circ}$ (for subject 1-6) in the $14851 \times 3$ dimensional shape space. The amount of variance explained by the PC are 34.7, 31.2, 18.3, 10.1 and $5.7 \%$. The full set of 31 mandibles are projected down into the subspace as shown in Fig. 2. The scores are shown using circles, crosses, pluses, squares, diamonds and triangles for the subjects 1-6. The loci of the grand mean pole (i.e. the origo) is marked by a star, and the loci of the patient specific mean shapes by labels $1-6$. Notice the lack of structure in the patient clustering indicating negligent 

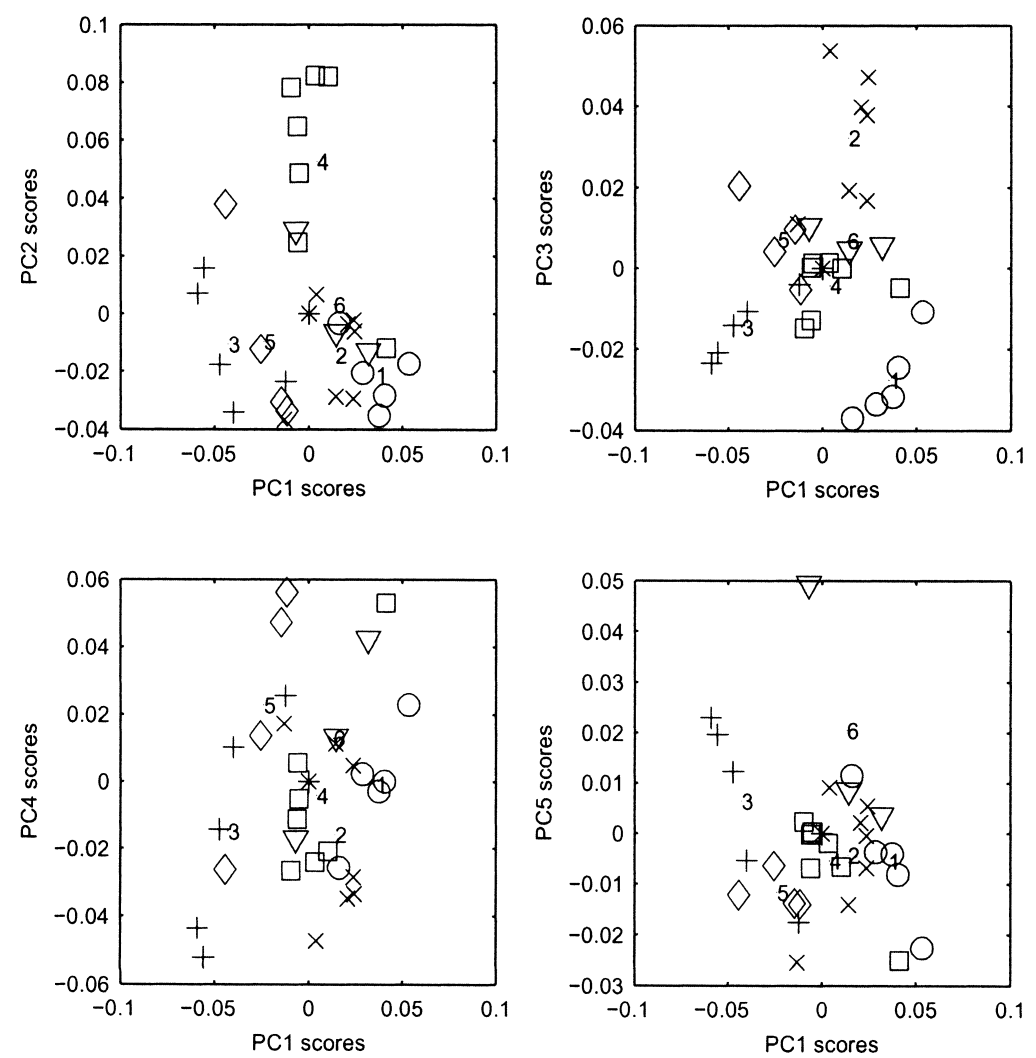

Fig. 2. Scores of the PC of the patient specific poles labeled 1-6. The * indicates the position of the grand pole. The scans of each patient $1-6$ are projected down into the five dimensional tangent space and are represented by $\bigcirc, \times,+, \diamond$ and $\nabla$ 's.

variation between the sexes of prepubescent subjects. As a consequence the sexes are pooled in the subsequent analyses. Also, notice the high degree of variation between subjects. The high degree of inter-patient variability thus leaves little room for variation related to the complexity of the shape if applying the grand pole for projecting into tangent space. Therefore, patient specific poles are applied when projecting into tangent space, where each mandible is corrected for the patient specific mean. The shape variability of each subject is thus centered on the grand pole of the Procrustes alignment, in effect removing the inter-patient variability.

The PC transform is applied to the mandibles projected into a 30 dimensional tangent space using patient specific poles. The first three PCs explain 62.2, 7.3 and 5.3\% of the total shape variation. In Fig. 3(a) we show pairwise scatterplots of: subject index, age, log age, centroid size (CS), and the first three PC modes. Notice the strong correlation between the PC1 and both CS and log age, of 0.826 and 0.861 , respectively. The correlation between CS and $\log$ age is very strong and amounts to 0.961. In (Andresen et al., 2000) similar results are presented and PC1 is applied to model mandibular growth on the basis of CS.

By parallel analysis (Horn, 1965), we have an objective manner of estimating the rank of the structured signal extracted from the tangent space by the decompositioning, see Fig. 4(left). The number of components that captures structured signal is determined by looking at the cutoff point of the observed eigenvalues, and of the eigenvalues of a PC analysis of unordered data (Buja and Eyuboglu, 1993). Over 100 permutation runs we consistently find four significant eigenmodes. However, to do prediction the modes must also correlate to mandibular growth. The scores versus size and age for the higher order PC components show low correlations (e.g. PC2-4 versus CS equals $0.09,-0.02,0.20$ ), see Fig. 3(a). Hence, in spite of the fact that the parallel analysis indicated the presence of significant structure in a higher dimensional space, modeling growth on the basis of a PC analysis is limited to a one-dimensional basis using only the first principal mode.

\subsection{Maximum autocorrelation factors analysis}

Orthogonality in Euclidean sense is a very limiting constraint when looking for interesting modes of biological variation. A more natural constraint is to require the resulting modes to be uncorrelated, thus allowing for non-Euclidean methods to be applied. Here we apply the MAF transform, which is a PC analysis in a metric space defined by the dispersion structure of spatially differenced landmarks.

Consider again the random signal variable $\boldsymbol{r}(\boldsymbol{x})$ and assume first and second order stationary. Let $\boldsymbol{\Delta}$ represent a 


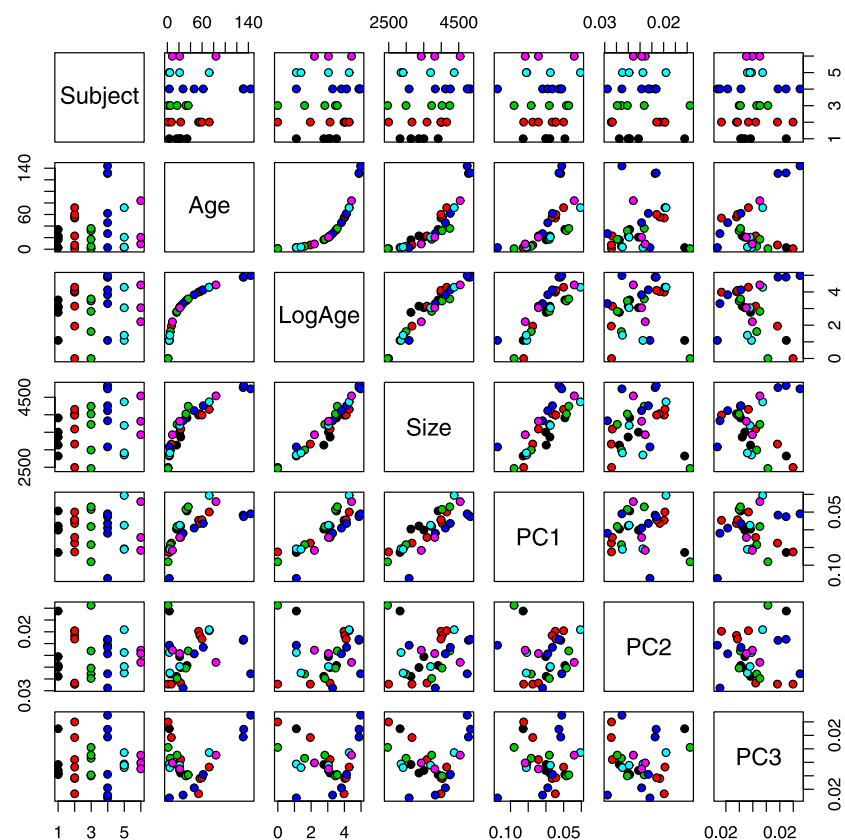

[(a) PCA]

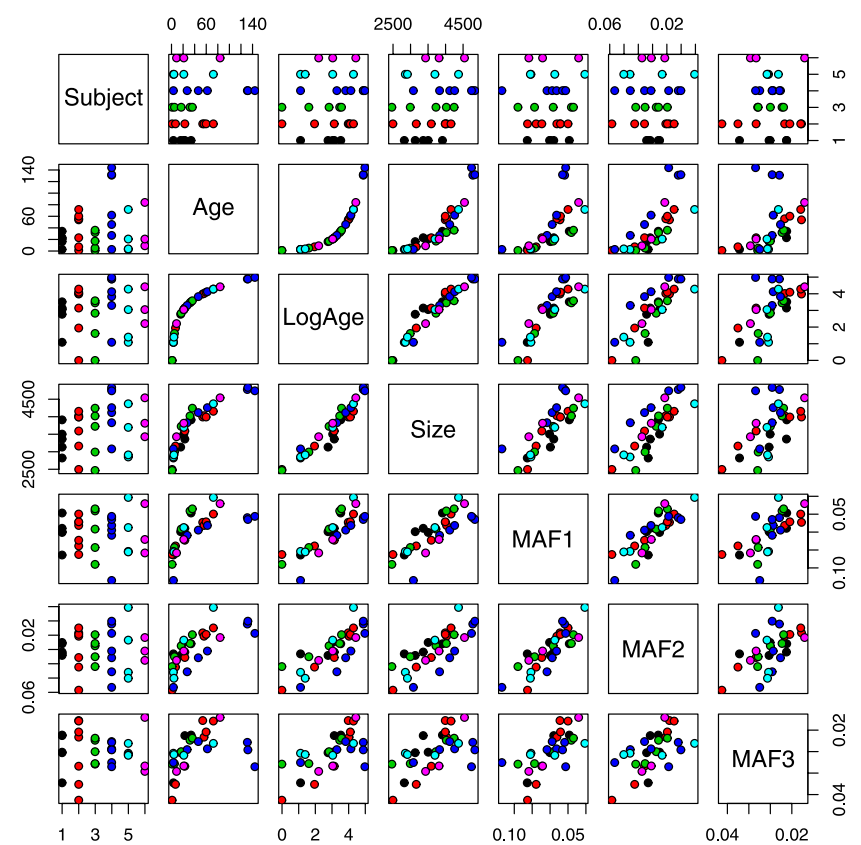

[(b) MAF]

Fig. 3. Pair-wise scatterplots of: subject, age, log age, CS, and the first three PCA and MAF modes, respectively.

spatial shift, then the spatial covariance function is defined by $\operatorname{Cov}\{\boldsymbol{r}(\boldsymbol{x}), \boldsymbol{r}(\boldsymbol{x}+\boldsymbol{\Delta})\}=\boldsymbol{\Gamma}(\boldsymbol{\Delta})$. Introducing $\boldsymbol{\Sigma}_{\boldsymbol{\Delta}}=D\{\boldsymbol{r}(\boldsymbol{x})-$ $\boldsymbol{r}(\boldsymbol{x}+\boldsymbol{\Delta})\}=2 \boldsymbol{\Sigma}-(\boldsymbol{\Gamma}(\boldsymbol{\Delta})+\boldsymbol{\Gamma}(-\boldsymbol{\Delta}))$, we find the autocorrelation expressed by

$\operatorname{Corr}\left\{\boldsymbol{a}_{i}^{\mathrm{T}} \boldsymbol{r}(\boldsymbol{x}), \boldsymbol{a}_{i}^{\mathrm{T}} \boldsymbol{r}(\boldsymbol{x}+\Delta)\right\}=1-\frac{1}{2} \frac{\boldsymbol{a}_{i}^{\mathrm{T}} \boldsymbol{\Sigma}_{\Delta} \boldsymbol{a}_{i}}{\boldsymbol{a}_{i}^{\mathrm{T}} \mathbf{\Sigma} \boldsymbol{a}_{i}}$

Let $\lambda_{1} \leqslant \cdots \leqslant \lambda_{P}$ be the eigenvalues of $\boldsymbol{\Sigma}_{\boldsymbol{\Delta}}$ with respect to $\boldsymbol{\Sigma}$ with the $a_{1}, \ldots, a_{P}$ corresponding conjugate eigenvectors. Then $z_{i}(\boldsymbol{x})$ is the $i$ th MAF. More specifically, we estimate $\boldsymbol{\Sigma}_{\boldsymbol{\Delta}}$ by the empirical dispersion structure of the
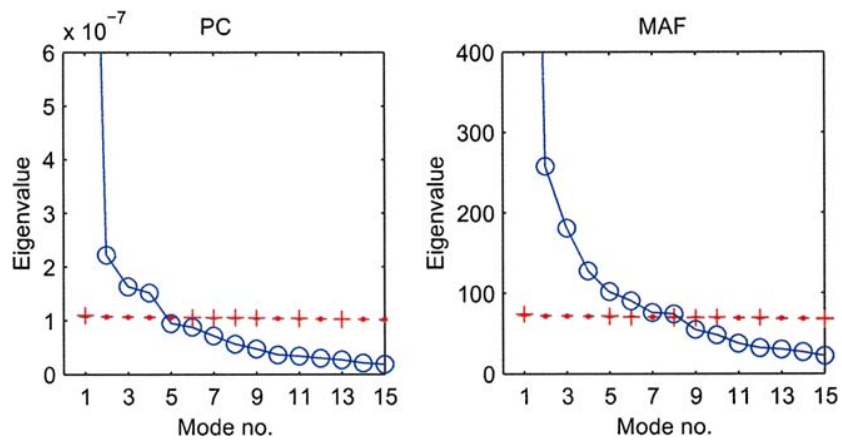

Fig. 4. Scree plot of the PC (left) and the MAF (right) analysis. Circles marks the analysis on the ordered data, and the boxplot summarizes analyses on 100 permutated data runs. The eigenvalues of the first PC and the first MAF mode not shown are $1.9 \mathrm{e}-6$ and 1600 , respectively. In the shape tangent space the PC analysis finds four significant modes, whereas the MAF analysis determines eight significant uncorrelated modes of variation. distances from each landmark to a plane, fitted in a least-squares sense to the neighboring point set.

The MAF transform can also be considered a Minimum Noise Fraction (MNF) transformation (Green et al., 1988), and thus maximizes the signal-to-noise ratio (SNR) in the new components. The SNR in the $i$ th component is then given by $2 \lambda_{i}-1$. Notice also that the MAF transform is equivalent to the independent component transformation proposed later by Molgedey and Schuster (1994). A formal proof is given in (Larsen et al., 2001; Larsen, 2002).

In Fig. 4(right) a scree plot is presented for the MAF analysis. We generalize the parallel analysis to the MAF problem by permutating the data that constitutes both $\mathbf{\Sigma}$ and $\Sigma_{\Delta}$. A consistent cut-off point is determined for all 100 permutation runs indicating that the first eight dominant MAF modes contain significant information. A more detailed decompositioning of the tangent space is thus obtained when compared with the results of the traditional PC analysis. In Fig. 3(b) we show pairwise scatterplots of the subject index, age, log age, CS, and the first three MAF modes. Notice how the MAF transform succeeds in finding multiple modes correlated with CS and log age. Particularly MAF1 and 2 show strong correlations to CS of 0.826 and 0.798. MAF3 has a correlation to CS of 0.579 . The correlations to log age are $0.860,0.822$ and 0.584 . Contrary to the higher order modes of the PC analysis, the second order MAF mode thus shows correlation to growth comparable to that of the principal modes, and can therefore, provide additional information to the growth modeling.

To compare the most interesting eigenmodes of the PC 
Table 2

Angles between eigenvectors correlated to growth derived from analyses on all the training data

\begin{tabular}{llll}
\hline & MAF1 & MAF2 & MAF3 \\
\hline PC1 & $3.7^{\circ}$ & $64.4^{\circ}$ & $75.8^{\circ}$ \\
MAF1 & & $65.5^{\circ}$ & $74.0^{\circ}$ \\
MAF2 & & & $82.3^{\circ}$ \\
\hline
\end{tabular}

and the MAF analysis, Table 2 includes the angles between the first three MAF eigenvectors (ignoring the sign), relative to each other, and to the first PC1 eigenvector. Notice the small angle between the principal modes of the PC and the MAF transforms. The methods show good agreement on the most important subspace of shape variability in the tangent space.

In Fig. 5 the first three MAF modes are shown. The red and blue mandibles represent deviations of, respectively, plus and minus two standard deviations (S.D.) of the total variability. Three different view-points are used.

The first order MAF appears to capture the primary growth pattern of the mandible. In particularly the vertical component of the mandibular growth including the eruption of the teeth is described. It is in good agreement with the most common direction of growth which results in a reduction of the condylar angles as shown. Due to the small angle between the PC1 and the MAF1 mode the above descriptions also applies to the variation related to the first order PC. The higher order MAF modes show less variability in terms of complexity of the shape, but may be used to explain lower order growth effects. The second order MAF focuses primarily on the transversal growth component, and appears also to include some aspect of asymmetry of the mandible. It captures the correlation between the backward sagital growth, the increase in condylar angles, and the forward tilting of the front teeth. It also detects correlation between the condylar growth direction and the concavity of the lower part of the mandible. The third MAF mode primarily relates to additional dynamics related to the eruption of the teeth and the transversal growth component.

\section{Shape prediction and evaluation}

Having established a better basis with anatomically plausible eigenmodes in the Procrustes tangent space we now turn to shape prediction and evaluation. The PC and the MAF models are applied in a leave-one-out study by excluding one patient at a time; $m=1, \ldots, 6$. For the MAF model we focus on the two most significant modes of variation with high correlations to size and age. In Table 3 we show some of the results of the cross-validation study. The angular deviations for the eigenvectors of the reduced sets are shown against PC1, MAF1 and MAF2 of the complete training set. Notice the good agreement of the orientation of the principal eigenvectors. This indicates

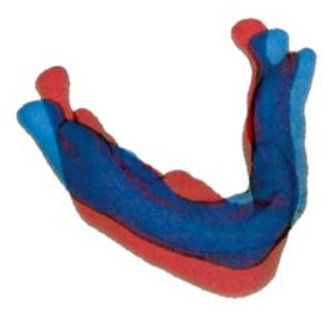

(a) MAF1

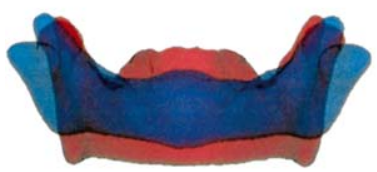

(d) MAF1

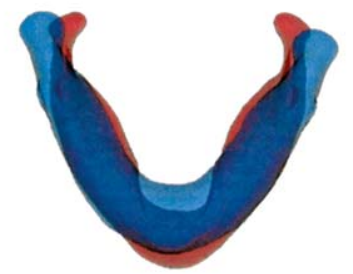

(g) MAF1

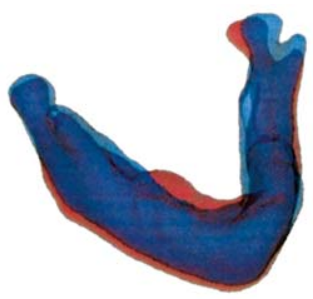

(b) MAF2

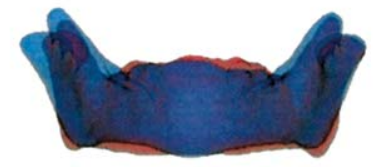

(e) MAF2

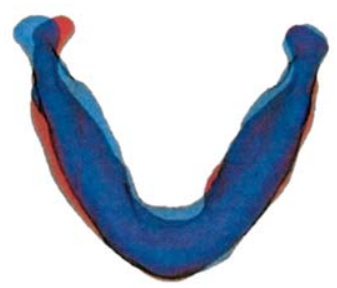

(h) MAF2

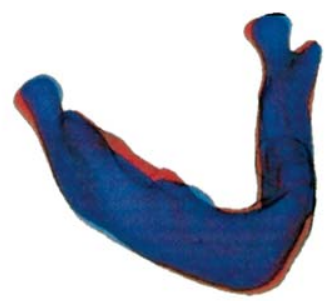

(c) MAF3

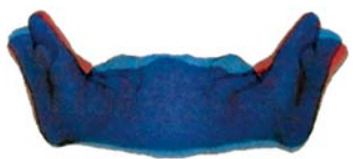

(f) MAF3

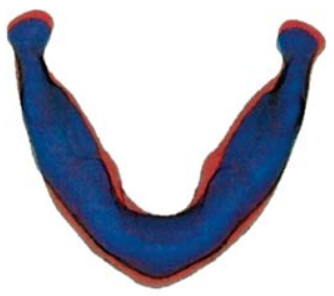

(i) MAF3

Fig. 5. Visualization of the three first MAF modes (column-wise) at \pm 2 S.D. from the mean mandible. The viewpoints are: oblique lateral, posterior and ventral view. 
Table 3

Cross-validation of the orientation of the eigenvectors from the reduced data sets against eigenvectors determined on the basis of all patients

\begin{tabular}{llll}
\hline Patient excluded & PC1 & MAF1 & MAF2 \\
\hline 1 & $4.1^{\circ}$ & $3.4^{\circ}$ & $9.7^{\circ}$ \\
2 & $5.8^{\circ}$ & $6.2^{\circ}$ & $38.4^{\circ}$ \\
3 & $6.5^{\circ}$ & $9.3^{\circ}$ & $38.7^{\circ}$ \\
4 & $8.3^{\circ}$ & $6.1^{\circ}$ & $32.9^{\circ}$ \\
5 & $5.9^{\circ}$ & $6.5^{\circ}$ & $16.9^{\circ}$ \\
6 & $3.9^{\circ}$ & $5.7^{\circ}$ & $32.5^{\circ}$ \\
\hline
\end{tabular}

that no patient controls the variability of the pooled analysis for neither the PC nor the, best, competing MAF transform. The stability of the analysis is primarily obtained by applying patient specific poles reducing the effect of inter-patient variability. Looking at the second MAF eigenvectors we notice an increase in the variability of the orientation, which is expected since it is a second order mode and the error accumulates.

When performing prediction new shapes can be generated on the basis of the model eigenvectors. The growth model is given by

$\boldsymbol{r}_{\text {future }}=\boldsymbol{r}_{i}+\boldsymbol{B}_{m} \boldsymbol{c}$, where $\boldsymbol{c}$ is a growth parameter vector and $\boldsymbol{B}_{m}$ contains the model basis in R-mode based on an analysis of the data set omitting the $m$ th patient. The model enables us to make prediction of a future shape, $\boldsymbol{r}_{\text {future }}$, for a new patient based only on a single CT scan, $\boldsymbol{r}_{i}$, registered to the common pole of the Procrustes alignment. Notice that although the model is linear in Procrustes space, it does not conflict with the findings that the growth appears non-linear in a biological coordinate system as described in (Björk and Skieller, 1983).

By varying $c$ the data analyst is able to examine the set of possible future shapes in the space spanned by the eigenvectors. If we want to make prediction in one specific direction of the subspace, the growth parameter becomes a scalar and can be estimated by regression on CS or the subject log age. The most common choice in other applications of Procrustes shape analysis is the CS and chosen as the independent variable in (Andresen et al., 2000) as well. The growth parameter is thus estimated by $c=\left(\mathrm{CS}_{\text {future }}-\right.$ $\left.\mathrm{CS}_{i}\right) \alpha$, where $\alpha$ is the regression coefficient determined from an analysis of the corresponding shape scores versus CS. In the scatterplots Fig. 3(a) and (b), we show the relationship between the scores of the different eigenvectors and CS. The regression coefficient is determined as the

Table 4

Prediction errors, distance between corresponding points ( $\mathrm{mm}$ ), of the cross-validation study

\begin{tabular}{|c|c|c|c|c|c|c|c|}
\hline \multirow{2}{*}{$\begin{array}{l}\text { Patient } \\
\text { number/scan/span }\end{array}$} & \multicolumn{2}{|l|}{ PC1 } & \multicolumn{2}{|c|}{ MAF1 } & \multicolumn{2}{|c|}{ MAF2 } & \multirow[t]{2}{*}{ Run \# } \\
\hline & Mean & S.D. & Mean & S.D. & Mean & S.D. & \\
\hline $1 / 1 / 3 \rightarrow 34$ & 2.4 & 1.3 & 2.4 & 1.3 & 4.3 & 1.4 & 1 \\
\hline $1 / 2 / 16 \rightarrow 34$ & 1.8 & 0.8 & 1.7 & 0.7 & 2.2 & 0.9 & 2 \\
\hline $1 / 3 / 21 \rightarrow 34$ & 1.8 & 0.7 & 1.7 & 0.7 & 2.3 & 0.9 & 3 \\
\hline $1 / 4 / 23 \rightarrow 34$ & 1.4 & 0.6 & 1.3 & 0.6 & 1.8 & 0.7 & 4 \\
\hline $2 / 1 / 1 \rightarrow 72$ & 3.5 & 1.4 & 3.4 & 1.4 & 4.3 & 1.9 & 5 \\
\hline $2 / 2 / 7 \rightarrow 72$ & 2.4 & 1.1 & 2.3 & 1.1 & 3.2 & 1.9 & 6 \\
\hline $2 / 3 / 23 \rightarrow 72$ & 2.3 & 1.3 & 2.2 & 1.3 & 3.0 & 1.8 & 7 \\
\hline $2 / 4 / 54 \rightarrow 72$ & 1.5 & 0.7 & 1.5 & 0.7 & 1.7 & 0.8 & 8 \\
\hline $2 / 5 / 56 \rightarrow 72$ & 1.6 & 0.8 & 1.6 & 0.8 & 1.8 & 0.8 & 9 \\
\hline $2 / 6 / 60 \rightarrow 72$ & 1.4 & 0.6 & 1.4 & 0.6 & 1.5 & 0.7 & 10 \\
\hline $3 / 1 / 1 \rightarrow 36$ & 2.8 & 1.3 & 2.8 & 1.3 & 5.4 & 2.2 & 11 \\
\hline $3 / 2 / 5 \rightarrow 36$ & 2.5 & 1.2 & 2.4 & 1.2 & 4.1 & 1.6 & 12 \\
\hline $3 / 3 / 17 \rightarrow 36$ & 2.1 & 0.9 & 2.1 & 0.9 & 2.7 & 1.2 & 13 \\
\hline $3 / 4 / 32 \rightarrow 36$ & 1.0 & 0.4 & 1.0 & 0.4 & 0.9 & 0.5 & 14 \\
\hline $4 / 1 / 3 \rightarrow 144$ & 3.7 & 1.6 & 3.6 & 1.6 & 7.2 & 2.6 & 15 \\
\hline $4 / 2 / 27 \rightarrow 144$ & 3.1 & 1.6 & 3.1 & 1.5 & 3.3 & 1.6 & 16 \\
\hline $4 / 3 / 46 \rightarrow 144$ & 2.8 & 1.4 & 2.8 & 1.4 & 3.0 & 1.4 & 17 \\
\hline $4 / 4 / 62 \rightarrow 144$ & 2.8 & 1.4 & 2.8 & 1.4 & 2.9 & 1.3 & 18 \\
\hline $4 / 5 / 131 \rightarrow 144$ & 2.0 & 1.0 & 2.0 & 1.0 & 2.0 & 1.0 & 19 \\
\hline $4 / 6 / 132 \rightarrow 144$ & 2.0 & 0.9 & 2.0 & 0.9 & 1.9 & 0.9 & 20 \\
\hline $5 / 1 / 3 \rightarrow 72$ & 2.6 & 1.0 & 2.7 & 1.1 & 5.5 & 2.1 & 21 \\
\hline $5 / 2 / 4 \rightarrow 72$ & 3.1 & 1.2 & 3.1 & 1.2 & 5.7 & 2.3 & 22 \\
\hline $5 / 3 / 21 \rightarrow 72$ & 2.1 & 1.0 & 2.1 & 1.0 & 3.4 & 1.5 & 23 \\
\hline $6 / 1 / 9 \rightarrow 84$ & 2.8 & 1.0 & 2.9 & 1.0 & 5.3 & 1.8 & 24 \\
\hline $6 / 2 / 21 \rightarrow 84$ & 2.8 & 1.2 & 2.8 & 1.2 & 4.2 & 1.7 & 25 \\
\hline
\end{tabular}

For decreasing time span (months) the predictions are improved. 


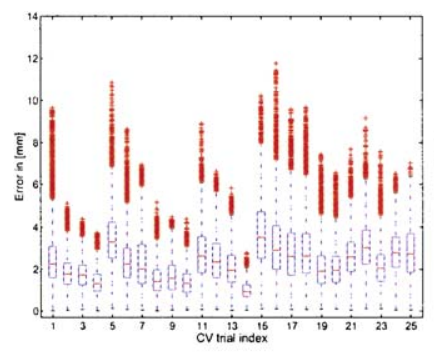

(a) $\mathrm{PC} 1$

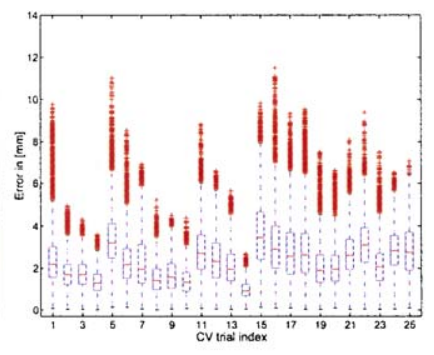

(b) MAF1

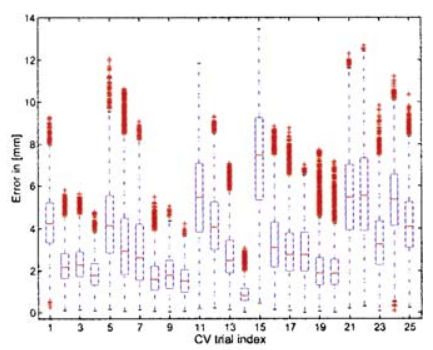

(c) MAF2

Fig. 6. Summary of the prediction studies for PC1, MAF1 and MAF2 based analyses by boxplots (top-down). For subject $1-6$ the 25 CV runs separate into the sets $1-4,5-10,11-14,15-20,21-23$ and $24-25$.

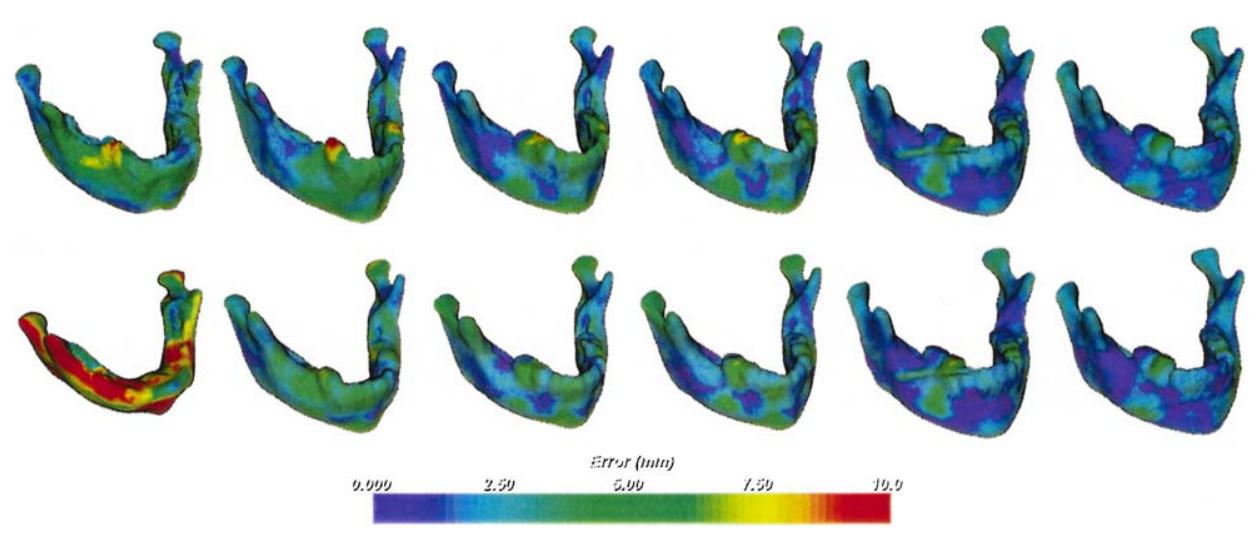

Fig. 7. Predicted future shapes based on the MAF1 (top) and the MAF2 (bottom) mode. The color coding shows the prediction error (mm). The accuracy increases for decreasing time span.

mean of the optimal regressions coefficients for each subject. This is done in order to limit the influence of patient variability and to improve robustness. The model in Eq. (2) so far only models shape variability, in order to include size the predicted shape must also be rescaled by $\mathrm{CS}_{\text {future }}$.

Table 4 shows the prediction errors of mandibular shape. For each patient, the oldest mandibular shape is predicted on the basis of its CS by each earlier form; using the PC and the MAF models based on the other five patients of the data set. The average error and deviation is shown for all experiments. For each run the best model has errors shown in bold, and the second best or competing models have errors shown in italic. Notice that the results for PC1 and MAF1 are very similar and gratifyingly homogeneous between subjects, and that the MAF analysis provides marginally better results for patient $1-4$. The ability to predict the most recent scan of patient 4 , at age 12 , on the basis of the first is rather noteworthy, since none of the other patients covers a similar large time span. The

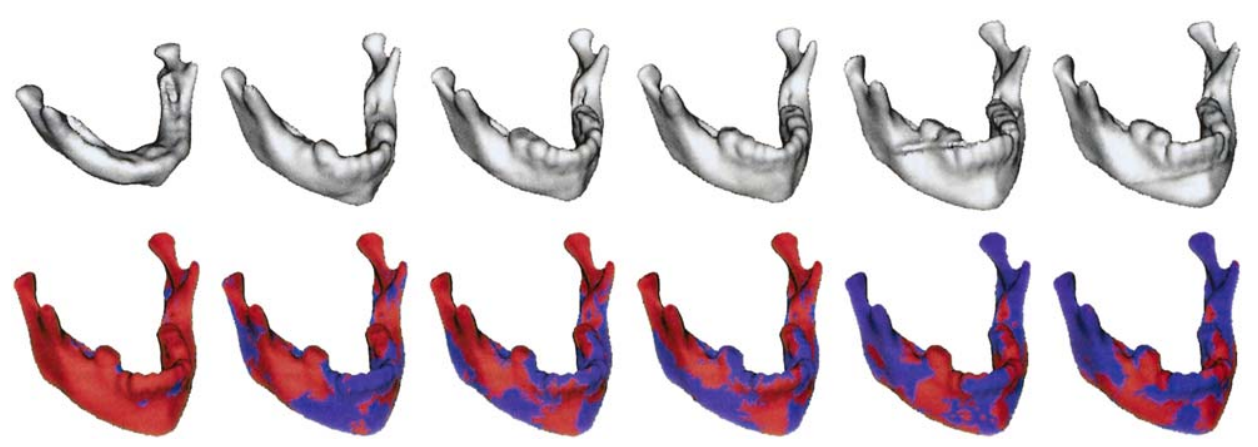

Fig. 8. Top row: The first 6 CT segmented mandibles, i.e. the source shapes, at 3, 27, 46, 62, 131 and 132 months, used in prediction study for patient 4. Bottom row: The target shape at 144 months with a color coding illustrating where MAF1 (red) is superior to MAF2 (blue) in the prediction. The dimensions of the 12-year-old target mandible are approximately 90,100 and $50 \mathrm{~mm}$. 
oldest subject contributing to the model in this case is only of age 7. Looking at the second order MAF mode the errors for predictions over large time spans are the worst. The mode thus seem to lack some of the dynamics that governs the initial growth of the human mandible and captured by MAF1. However, predicting the future shape based on more recent scans the results of MAF2 becomes comparable to those of PC1 and MAF1, see subject 1-4. The mode thus appears to explain variation in mandibular shape present after the initial growth of the mandible dominated by a strong vertical component which decreases with age (apparently up to about 25-30 months). The failure to model patient 5 and 6 successfully for the MAF2 mode is thus partly explained due to relative early initial scans of the subjects.

The cross-validation runs shown in Table 4 are also reported in Fig. 6 providing a more detailed picture on the distribution of the prediction errors for the PC and the MAF modes. The prediction error is measured by the distance between corresponding points. The total of 25 trial runs are concatenated and labeled 1 through 25 . Notice the decreasing number of outliers in the boxplots when applying more recent scans in the prediction.

In Fig. 7 the predicted shapes of patient 4 at 144 months are shown using different models, and can be compared with the boxplots labeled 15-20. Results are shown applying the MAF1 and the MAF2 mode of biological variation. Notice that for decreasing time span the predicted shapes becomes more accurate. In Fig. 8 the initial CT scans, i.e. the sources for the predictions and the target mandible are shown. The red regions on the target mandible indicates where MAF1 performs better than MAF2 in the prediction. Notice that the MAF2 modes lacks the vertical component which makes MAF1 able to perform reasonably when applying the oldest CT scan. Looking at the subsequent predictions the difference in the growth patterns of MAF1 and MAF2 is primarily observed in the transversal plane and in the configuration of the teeth. For the most recent scans the difference in the distribution of the error between the MAF1 and MAF2 models is reduced to an average of $0.08 \pm 0.19 \mathrm{~mm}$ S.D., with MAF1 having more than $85.2 \%$ of the errors less than $3 \mathrm{~mm}$ and MAF2 more that $88.3 \%$.

It is important to stress, that the data analyst can examine the future shape prediction by smooth transitions between the MAF1 and MAF2 shape hypothesis. In practice the best prediction is often found at an intermediate value of these two eigenmodes.

\section{Conclusion}

By modeling the shape variability of the human mandible in Procrustes space we obtain linear growth models which produce accurate predictions of future shapes in our leave-one-out studies.

In building the shape model, the analysis on subject specific poles shows the need for removing the interpatient variability before modeling the mandible shape. The shapes are thus centered on the grand pole of the Generalized Procrustes analysis. Moreover, the inter-patient study revealed no structure in gender differences of prepubescent subjects, thus allowing for a pooling of the entire training set.

Analyses of the shape variability is performed using both the PC and the MAF transform. The novelty consists of applying the latter transform to produce a decompositioning of the modes of variation in a non-Euclidean metric, maximizing the SNR. The MAF transform decomposes the data into spatially coherent signals and tends to capture growth in a more intuitive and interpretable way than the PC analysis which does not take the spatial layout of the data into account. However, there may be some bias in the presented results since they are based on relatively sparse data. In contrast to the PC analysis, the new approach is invariant to linear transformations such as scaling of the individual components in the training set. As a consequence, the MAF analysis offers an elegant manner of handling size and scale that dominates the PC analysis by its construction.

By construction the MAF transform finds multiple eigensolutions representing uncorrelated modes of biological variation. Applying the non-Euclidean metric for shape decompositioning results in, not just a one dimensional time line of mandible shape evolution as detected by the traditional PC analysis, but spans a plane producing a set of possible configurations of the mandible at a given future time instance. Two one-dimensional traces in the solution plane are examined, namely the directions of the first two uncorrelated eigenmodes. The prime result of the leave-one-out prediction study, shows that the first MAF mode represents the dominant growth effects of the mandible, whereas the second order MAF mode included dynamics that primarily affect the mandible after the initial predominant vertical growth component has declined.

\section{Acknowledgements}

The authors would like to thank Dr Sven Kreiborg (School of Dentistry, University of Copenhagen, Denmark) and Dr Jeffrey L. Marsh (Department for Pediatric Plastic Surgery, Washington University School of Medicine at St. Louis Children's Hospital, St. Louis, Missouri, USA) for providing the CT scans, and Dr Per R. Andresen (formerly IMM, DTU) for the work done on the initial segmentation and registration of the data. The work was supported by the Danish Technical Research Council under grant number 26-01-0198 which is hereby gratefully acknowledged. 


\section{References}

Andresen, P.R., Nielsen, M., 2001. Non-rigid registration by geometryconstrained diffusion. Medical Image Analysis 5 (2), 81-88.

Andresen, P.R., Bookstein, F.L., Conradsen, K., Ersbøll, B.K., Marsh, J., Kreiborg, S., 2000. Surface-bounded growth modeling applied to human mandibles. IEEE Transactions on Medical Imaging 19 (11), 1053-1063.

Björk, A., Skieller, V., 1983. Normal and abnormal growth of the mandible. A synthesis of longitudinal cephalometric implant studies over a period of 25 years. European Journal of Orthodontics 5, 1-46.

Buja, A., Eyuboglu, N., 1993. Remarks on parallel analysis. Multivariate Behavioral Research 27 (4), 509-540.

Conradsen, K., Nielsen, B.K., Thyrsted, T., 1985. A comparison of $\min / \max$ autocorrelation factor analysis and ordinary factor analysis. In: Proceedings from Symposium in Applied Statistics, Lyngby, Denmark, pp. 47-56.

Davies, R.H., Twining, C.J., Cootes, T.F., Waterton, J.C., Taylor, C.J., 2002. 3D statistical shape models using direct optimisation of description length. In: Proceedings of the ECCV, Vol. 3, pp. 3-20.

Dryden, I.L., Mardia, K.V., 1998. Statistical Shape Analysis. Wiley, Chichester.

Goodall, C., 1991. Procrustes methods in the statistical analysis of shape. Journal of the Royal Statistical Society, Series B 53 (2), 285-339.

Green, A.A., Berman, M., Switzer, P., Craig, M.D., 1988. Transformation for ordering multispectral data in terms of image quality with implications for noise removal. IEEE Transactions on Geoscience and Remote Sensing 26 (1), 65-74.

Hilger, K.B., 2001. Exploratory analysis of multivariate data, unsupervised image segmentation and data driven linear and non-linear decomposition, Ph.D. thesis, Informatics and Mathematical Modelling, Technical University of Denmark, Kgs. Lyngby, 186 pp.

Hilger, K.B., Stegmann, M.B., Larsen, R., 2002. A noise robust statistical texture model. In: Medical Image Computing and Computer-Assisted Intervention - MICCAI 2002, 5th International Conference, Tokyo, Japan, vol. 2, pp. 444-451.
Horn, J.L., 1965. A rationale and test for the number of factors in factor analysis. Psychometrika 30, 179-186.

Hotelling, H., 1933. Analysis of a complex of statistical variables into principal components. Journal of Educational Psychology 24, 417441.

Johnson, R.M., 1963. On the theorem stated by Eckart and Young. Psychometrika 28, 259-263.

Kreiborg, S., Aduss, H., Cohen, M.M. Jr, 1999. Cephalometric study of the Apert syndrome in adolescence and adulthood. Journal of Craniofacial Genetics Development Biology 19, 1-11.

Larsen, R., 2002. Decomposition using maximum autocorrelation factors. Journal of Chemometrics 16 (8-10), 427-435.

Larsen, R., Eiriksson, H., Stegmann, M.B., 2001. Q-MAF shape decomposition. In: Medical Image Computing and Computer-Assisted Intervention - MICCAI 2001, 4th International Conference, Utrecht, The Netherlands, Vol. 2208 of Lecture Notes in Computer Science, Springer, pp. 837-844.

Larsen, R., Hilger, K.B., Wrobel, M.C., 2002. Statistical 2d and 3d shape analysis using non-Euclidean metrics. In: Medical Image Computing and Computer-Assisted Intervention - MICCAI 2002, 5th International Conference, Tokyo, Japan, Vol. 2488 of Lecture Notes in Computer Science, Springer, pp. 428-435.

Molgedey, L., Schuster, H.G., 1994. Separation of a mixture of independent signals using time delayed correlations. Physical Review Letters 72 (23), 3634-3637.

Paulsen, R.R., Hilger, K., 2003. Shape modeling using markov random field restoration of point correspondences. In: Information Processing in Medical Imaging, IPMI.

Switzer, P., Green, A.A.,1984. Min/max autocorrelation factors for multivariate spatial imagery, Techinal Report 6, Department of statistics, Stanford University.

Switzer, P., Ingebritsen, S.E., 1986. Ordering of time-difference data from multispectral imagery. Remote Sensing of Environment 20, 85-94.

ten Berge, J.M.F., 1977. Orthogonal Procrustes rotation for two or more matrices. Psychometrika 42, 267-276. 